\title{
APLICAÇÃO DA TERAPIA COGNITIVO - COMPORTAMENTAL SOBRE OS EFEITOS DO ESTRESSE NO SERVIDOR DE SEGURANÇA PÚBLICA
}

\section{APPLICATION OF COGNITIVE - BEHAVIORAL THERAPY ON THE EFFECTS OF STRESS ON THE PUBLIC SAFETY SERVER}

\section{Mônica de Lima Lopes ${ }^{1}$}

RESUMO: Este Artigo vem abordar a aplicação da Terapia Cognitivo - Comportamental, face às incidências e efeitos do estresse nos profissionais de Segurança Pública, sendo este considerado um dos principais riscos ocupacionais existentes no ambiente de trabalho, causadores de danos e ou agravos à saúde destes profissionais. Neste contexto, tem-se como objetivo principal evidenciar a importância da prevenção, tratamento e acompanhamento dos policiais e seus familiares, incluindo a promoção da saúde, de qualidade de vida e bem-estar no exercício de sua função. Desta forma, foi utilizado como metodologia de trabalho um estudo de análise exploratória e bibliográfica do tipo descritiva. Observou-se que atualmente os princípios e as metas de necessidade da valorização dos profissionais, na tentativa de reduzir os riscos de adoecimento no desempenho de suas funções, buscam o modelo biopsicossocial entre as dimensões biológicas, psicológicas e sociais. Mesmo com o ambiente de trabalho inadequado, muitas vezes o policial precisa adaptar-se, contudo, no decorrer dos anos, as doenças ocupacionais começam a surgir. Como maior consequência, observamos a sobrecarga física e emocional, ocasionadas pelo acumulo de estresse gerado por um ambiente hostil e dificuldade de enfrentamento diante de situações difíceis que exige do profissional, um nível de atenção elevada. Diante do contexto, acredita-se que é necessário maior investimento na qualidade de vida do policial, através de ações de saúde, mudança de cultura institucional e valorização do profissional influenciando o bem-estar, dando continuidade aos trabalhos e otimização das atividades dos Servidores de Segurança Pública.

Palavras-chave: Estresse, Segurança Pública, Terapia Cognitivo-Comportamental.
ABSTRACT: This Article is addressing the application of Cognitive - Behavioral Therapy, given the incidences and effects of stress on public safety professionals, which is considered one of the existing occupational hazards in the workplace, causing damage and or health problems these professionals. In this context, it has as main objective to highlight the importance of prevention, treatment and monitoring of police officers and their families, including health promotion, quality of life and well-being in the exercise of its function. Thus, it was used as a working methodology a study of exploratory and descriptive analysis of the literature. It was noted that currently the principles and the need for enhancement of professional goals in an attempt to reduce the risk of illness in the performance of its functions, seek the biopsychosocial model between the biological, psychological and social dimensions. Even with inadequate work environment, often the police need to adapt, however, over the years, the occupational diseases begin to emerge. As greater consequence, we observe the physical and emotional burden, caused by the accumulation of stress generated by a hostile environment and difficulty coping in difficult situations requiring professional, high a level of attention. On the context, it is believed that it is necessary to invest more in the police quality of life through programs in health, institutional culture change and enhancing the professional influencing the wellbeing, continuing the work and optimization of the Security Servers activities public.

Keywords: Stress, Public Security, Cognitive-Behavioral Therapy. 


\section{INTRODUÇÃO}

O estresse, palavra de origem inglesa, é utilizado, segundo Hans Selye (1984), para designar o estado em que se encontra um organismo ameaçado de desequilíbrio. Serve para estabelecer o equilíbrio interior $o$ qual sofre modificações pelas diferentes exigências exteriores. Trata-se de uma síndrome conhecida por Síndrome Geral de Adaptação, que se desenvolve em três fases: fase de alerta, fase de resistência e fase de exaustão (LIPP, 1996).

O estresse não é uma doença, mas uma preparação do organismo em resposta a um novo estímulo, ou seja, uma reação biológica essencial para adaptação a novas situações, a qual varia de uma pessoa para outra. $O$ estresse não se refere somente a situações negativas, apesar de ser uma definição utilizada nos momentos de tensão, é o conceito usado ao conjunto de reações do organismo quando se depara com qualquer estímulo, tanto ameaçador que incita desconforto, como uma expectativa favorável (MICHAELA, 2011).

O ambiente organizacional pode ser considerado uma das fontes promotoras de estresse, chamado estresse ocupacional. É produto da relação entre o indivíduo e o seu ambiente de trabalho, em que as exigências deste ultrapassam as habilidades do trabalhador para enfrentá-las, o que pode desencadear um desgaste excessivo do organismo, interferindo na sua produtividade (DEJOURS, 1990).

\section{Coman e Evans}

(1991) investigaram reações físicas dos policiais em relação a conteúdo de trabalho e fatores de contexto de trabalho. Os autores encontraram, como fatores estressantes de trabalho, aqueles relacionados às atividades executadas pela polícia enquanto lidando com o público, provendo um serviço público ou prendendo criminosos. Também detectaram fatores do contexto de trabalho relacionados com os fatores organizacionais e fatores de rotina profissional, como promoção e procedimentos disciplinares e papel do trabalho, sendo que 0 fator mais estressante foi lidar com situações perigosas e violentas ou com o resultado das mesmas.

Albrecht (1988) faz referência a um grupo de fatores estressantes no ambiente do trabalho que pode afetar a qualidade de vida profissional dividindo-os em físicos, sociais e emocionais. Dentre os fatores físicos, o autor se refere a temperatura, barulho, vibração, poluidores do ar, lesões físicas, máquinas perigosas, animais perigosos e substâncias potencialmente explosivas ou tóxicas. Entre os fatores sociais estão: chefe, colegas de trabalho, clientes e outras pessoas com as quais o relacionamento represente risco de danos morais ou pessoais. Quanto aos fatores emocionais, há: prazos, risco percebido de lesão física, risco financeiro pessoal, necessidade de prestação de contas por tarefas de alto risco, medo de perder status, expectativa de fracasso e de desaprovação de outras pessoas importantes.

O exercício profissional das forças de segurança é considerado uma das ocupações mais estressantes do mundo. Os motivos para esta classificação 
compreendem-se com a natureza das funções prestadas onde, para além dos problemas mais comuns a outras ocupações, trabalho por turnos, excesso de horas de trabalho, etc. (DANTZER, 1987; SELYE, 1984).

\section{JUSTIFICATIVA}

$\mathrm{O}$ interesse pelo tema surgiu com ênfase às questões relacionadas às condições adversas de trabalho, jornadas e riscos da profissão, que entende-se como surgimento de doenças ocupacionais e altos índices de estresse e desgaste emocional, físico e psíquico. A exploração teórica justifica-se em buscar estratégias de identificação e atuação segundo as técnicas utilizadas na terapia cognitivo-comportamental, como meio de enfrentamento e ajustamento à satisfação no exercício da profissão, investir no profissional, e valorizar seu trabalho, além de prover apoio e atenção aos interesses e dificuldades pessoais que estejam enfrentando. Contudo, surge a necessidade de criar um programa específico voltado para a qualidade de vida do trabalhador.

Sendo assim, esse estudo tem como objetivo geral identificar a incidência de estresse no servidor de Segurança Pública, no que se refere aos aspectos promotores de estresse e saúde ocupacional. Pretende-se também:

- Identificar a incidência de estresse no servidor de Segurança Pública;

- Investigar as estratégias defensivas utilizadas frente às situações estressoras;

- Averiguar os aspectos relativos à organização do trabalho dos sujeitos;

\section{MÉTODO}

$\mathrm{Na}$ tentativa de aprender os objetivos propostos, foi lançado mão de um estudo exploratório do tipo bibliográfico, de método qualitativo. O enfoque literário visou compreender o fenômeno proposto através de instrumentos como, artigos científicos, livros como bases de referências e artigos de revistas.

A revisão bibliográfica consiste em uma pesquisa diferenciando-se de levantamento de dados, na qual busca-se informações disponíveis em publicações como livros teses e artigos de renome nacional ou não em páginas da web feitos por autores diversos (Rodrigues, 2007).

\section{RESULTADOS/DISCUSSÃO}

Lipp (2014) descreve o estresse como uma reação do organismo com componentes psicológicos, físicos, mentais e hormonais que ocorrem quando surge a necessidade de uma adaptação grande a um evento ou situação de importância, podendo ser positivo ou negativo.

De acordo com Selye (apud Lipp 1996), o estresse conhecido por síndrome geral de adaptação, se desenvolve em três fases: fase de alerta, fase de resistência e fase de exaustão. A fase de alerta inicia-se quando a pessoa se confronta com o estressor pela primeira vez. Neste momento, uma reação de alerta se instala e o organismo se prepara para a luta ou para a fuga com a consequente quebra da homeostase. Quando o estressor tem uma duração curta à adrenalina é eliminada e ocorre a restauração da homeostase, e a pessoa sai dessa fase sem complicações para o seu bem-estar. É nessa fase que ocorre um aumento na produtividade, se a pessoa sabe administrar o estresse, ela pode utilizá-lo em seu benefício devido à 
motivação, entusiasmo e energia que esta fase produz.

Segundo Lipp e Malagris (1995), dois sintomas aparecem com bastante frequência nesta fase: a sensação de desgaste generalizado sem causa aparente e dificuldade com a memória. Na terceira fase, a da exaustão, conforme Selye apud Lipp (1996), o estresse já se tornou intenso demais, devido a pessoa não ter energias suficientes para lidar com 0 estressor ou devido a outros estressores que ocorrem concomitantemente. A combinação de vulnerabilidade individual com estímulos com potencial estressor leva ao desenvolvimento de estresse, o qual se manifesta tanto ao nível individual quanto ao organizacional.

Do ponto de vista individual, França e Rodrigues (1997), relaciona como principais sintomas de estresse: nervosismo, ansiedade, irritabilidade, fadiga, sentimentos de raiva, angústia, período de depressão, dor no estômago, dor nos músculos do pescoço e ombros e dores discretas no peito quando o indivíduo se encontra sob pressão.

As manifestações habituais podem ser mentais e/ou cognitivas (sentimentos de desamparo, fracasso e impotência; baixa auto-estima; inquietude e dificuldade para concentração;

comportamentos paranóicos e/ou agressivos com companheiros e família); físicas (cansaço; dores osteo-articulares e cefaléias; transtornos do sono; alterações gastrointestinais, taquicardias); de conduta (consumo elevado de café, álcool, fármacos e drogas ilegais; absenteísmo no trabalho; baixo rendimento pessoal; conflitos interpessoais no trabalho e no ambiente familiar) (GIL, 2002 apud
FREITAS, 2010).

Segundo Dejours (1992), a primeira vítima do trabalho inseguro não é o aparelho psíquico; mas, sim, o corpo que se entrega na execução das atividades laborais e acaba adquirindo complicações inerentes do local de trabalho precarizado; dessa forma, têm-se um trabalhador fragilizado, e arriscando a vida, para cumprir as obrigações prescritas pela organização.

Dejours (1992) aponta para elementos que auxiliam na compreensão das condições e organização do trabalho e da vivência dos trabalhadores que nela desenvolvem suas atividades e aí passam grande parte de sua vida. Chama a atenção para a organização do trabalho, que, segundo o ele, é a principal responsável pelo surgimento de experiências danosas ou não ao psiquismo do trabalhador.

Dificuldades em conciliar de forma satisfatória a vida familiar e ocupacional estão entre os fatores associados ao estresse no Servidor de Segurança Pública. Estudos demonstram que a associação trabalho-família possui reflexos positivos e negativos no bemestar das pessoas, sendo que em um momento de crise eles podem amparar e proteger o sujeito, como também podem ser um gatilho para o adoecimento (HALBESLEBEN e ZELLARS, 2010).

Halbesleben e Zellars (2010) apontam que muitas vezes o conflito relacionado à indisponibilidade familiar e desequilíbrio na vivência desses dois papéis (trabalhador-integrante de um grupo familiar), criam tensões e cargas psíquicas intensas que podem levar o 
trabalhador ao adoecimento, pois o mesmo não consegue lidar sozinho com as pressões que estão lhe confrontando.

\section{RELAÇÃO SAÚdE E ADOECIMENTO}

Atualmente, alguns autores (GilMonte, 2005; Kalimo, El-Batawi \& Cooper, 1988; Lipp, 1996) questionam se o estresse derivado da realização do trabalho é uma das principais causas de enfermidade laboral, de absenteísmo e da origem de muitos acidentes. Gil-Monte (2005) afirma que a prevenção dos riscos psicossociais no trabalho que podem gerar estresse e a prevenção dos acidentes laborais têm tido um grande destaque nos últimos tempos.

Halbesleben e Zellars (2010) relatam que uma das causas do conflito entre os papéis trabalho-família refere-se ao tempo, ou seja, quando a pessoa se entrega a um dos papéis fisicamente, quando permanece afastado de um dos ambientes (trabalho ou família). Quanto ao psicológico, a subjetividade (pensamentos, fantasias, etc.) do mesmo está focalizada em apenas um dos locais. Os autores também inserem o conceito de Spillover que pode ser identificado quando "um campo passa a ter influência (de maneira positiva ou negativa) sobre o outro, ou seja, uma situação estressora em uma área da vida mobilizará todas as outras"(HALBESLEBEN e ZELLARS, 2010, P.60).

\section{Halbesleben e Zellars (2010)} descrevem que quando os profissionais precisam encontrar estratégias para 0 enfrentamento do estresse ou do conflito trabalho-família, atenuado com o sentimento de impossibilidade de cumprir sua tarefa. A tendência é de que eles não consigam reduzi-lo, esta situação se inverte quando a organização do trabalho investe em ações sistêmicas mais amplas para prevenir e amparar seus colaboradores.

\section{A TERAPIA COGNITIVO- COMPORTAMENTAL APLICADA NO ENFRENTAMENTO DO ESTRESSE}

A Terapia Cognitivo-Comportamental, conhecida como TCC, utiliza técnicas e conceitos vindos de duas principais abordagens, sendo estas a cognitiva e a comportamental. Seu conceito tem uma estrutura "biopsicossocial” na determinação e compreensão dos fenômenos relativos a psicologia humana, constituindo-se como uma abordagem que focaliza o trabalho sobre os fatores cognitivos da psicopatologia (BAHLS e NAVOLAR, 2004).

Segundo Beck (1997) a TCC basear-se numa abordagem estruturada, diretiva, ligada a uma relação genuína entre paciente e terapeuta, pautada pela empatia e colaboração mútua. Seu foco principal é a psicoeducação, as técnicas utilizadas visam identificar, testar a realidade e corrigir as conceituações cognitivas distorcidas.

De acordo com Corrêa e Menezes (2002), o estresse negativo tem afetado um número crescente de trabalhadores em todo o mundo, provocando, além de sofrimento físico e mental, prejuízos financeiros também crescentes às empresas e ao sistema de previdência e seguridade social. Estudos confirmam a importância do estresse nas doenças do coração, de pele, gastrintestinais, neurológicas e em distúrbios ligados ao sistema imunológico e emocional. 
Conforme proposto por Lipp (1996) no treino de controle do estresse, o tratamento do estresse, precisa aliar as técnicas cognitivas intervenções mais específicas (como exercícios de relaxamento) à prática regular de exercícios físicos e uma nutrição adequada. No caso do tratamento do estresse a hipnose principal do papel do exercício é sua função ativadora da liberação de betaendorfinas responsáveis pelas sensações de conforto, prazer, alegria e bem estar.

Hart, Wearing e Headey (1995) sugerem que, para desenvolver projetos de intervenção no sentido de reduzir o estresse de policiais, deve ser adotada uma abordagem mais sistêmica, em razão do complexo relacionamento de variáveis que explicam o bem-estar psicológico desses trabalhadores. O essencial, de acordo com França e Rodrigues (1997), seria melhorar a qualidade da rotina de trabalho. Segundo esses autores, deve-se valorizar mais o próprio corpo e estar alerta para os sintomas que se repetem, como dor de cabeça e insônia, antes que se adoeça.

\section{CONSIDERAÇÕES FINAIS}

Diante do contexto apresentado, observa-se que os objetivos foram atingidos uma vez que na profissão em questão, faz-se necessário investir no profissional e valorizar o seu trabalho, tomando como base o tipo de atividade e situações que estes grupos enfrentam cotidianamente, que eventualmente os expõe a riscos de vida e integridade física. Promover apoio e atenção aos interesses e dificuldades pessoais que estejam enfrentando, em consequência das diversas influências causadoras do estresse e que podem ser danosas ou não a saúde física e mental do policial. Este profissional, portanto, se torna sujeito a pressões causadoras de sofrimento psíquico, o que sugere a necessidade de um suporte preventivo e terapêutico nos quadros que já se encontram instalados.

Entende-se que a autoexigência do profissional ou até mesmo situações em que seu grau de profissionalismo é colocado em questão negativamente, torna o indivíduo propenso a níveis de estresse altíssimos pelo conflito vivido no seu dia a dia. O desinteresse por outras atividades, principalmente atividades físicas, são em percentual elevado dentro das corporações policiais, que abre grande precedente para problemas associados ao estresse como o uso abusivo de substâncias psicoativas.

Conclui-se, portanto, que o Estresse pode ser controlado através de Técnicas Psicoterápicas desenvolvidas através da TCC, visando promover o mais realista tanto da demanda do ambiente e de sua importância, quanto das capacidades do indivíduo, de modo a gerar apenas ativação necessária dos mecanismos de estresse.

Sendo assim, necessita-se de mais estudos na área e cabe ao Terapeuta Cognitivo - Comportamental estar atento e chamar a atenção do paciente para o repentino aumento do nível de energia, incompatível com a condição física anterior, trabalhando em termos psicocognitivos e psicoeducativos. O terapeuta tem como fundamental importância do seu trabalho indicar a prática de exercícios físicos como parâmetro de qualidade de vida e controlador do estresse. 


\section{REFERÊNCIAS}

ALBRECHT, K. (1988). O gerente e o estresse. Rio de Janeiro: Zahar.

BAHIS, Saint-Clair; NAVOLAR, Ariana Bassetti Borba. Terapia Cognitivo-Comportamentais: Conceitos e Pressupostos Teóricos. Revista eletrônica de Psicologia. n. 04, Curitiba, jul. 2004 Disponível em www.utp.br/psico.utp.online

BECK. Judith S.; Terapia Cognitiva: teoria e prática. Porto Alegre: Artes Médicas, 1997.

COMAN, G. \& Evans, B. (1991). Stressors facing the Australian police in the 1990s. Police Studies, 14, 153-65.

CORRÊA, Sebastião Augusto; MENEZES, José Ricardo de Melo. Estresse e trabalho. Campo Grande, MS, 2002.

DANTZER, M. L. (1987). Police related stress: A critique for future research._Journal of Police and Criminal Psychology, 3,_43-48.

DEJOURS. C. A loucura do Trabalho. São Paulo, SP, 1992.

FRANÇA, A. C. L. \& Rodrigues, A. L. (1997)._Stress e trabalho: guia básico com abordagem psicossomática. São Paulo: Atlas.

FREITAS, Ângela Maria de. Impacto do estresse cotidiano no funcionamento executivo de Policiais Civis do RS. Porto Alegre: PUCRS, 2010.

Disponível em <http:www.forumsegurança.org.br> Acesso em: 14 de outubro de 2011. O manejo do stress In: RANGE, B. (org).

Psicoterapia Comportamental e Cognitiva: pesquisa prática, aplicações e problemas. Campinas: Fundo Editorial Psy, 1995.

GIL-MONTE, P. R. (2005). Factorial validity of the Maslach Burnout Inventory (MBI-HSS) among Spanish professionals._Revista Saúde Pública, 39(1), 23-35.

HALBESLEBEN, J. R. B.; ZELLARS, K. L. Stress e a Interface trabalho-família. In: Rossi, Ana Maria Rossi; PERREWÉ, Pamela L.; SAUTER, Steven L. (Orgs.). Stress e qualidade de vida no trabalho: Perspectivas atuais da saúde ocupacional.1 ed. São Paulo: Atlas, 2010, p.56-72.

HART, P. M., Wearing, A. J. \& Headey, B. (1995).

Police stress and well-being: Integrating personality, coping and daily work experiences._Journal of Occupational and Organizational Psychology, 68, 133-156.
(1988)._Los factores psicosociales en el trabajo y su relación con la salud. Ginebra: Organización Mundial de la Salud.

LIPP, Marilda Novais (org.). Pesquisa sobre Estresse no Brasil: saúde, ocupação e grupo de risco. São Paulo: Papirus, 1996.

LIPP, Marilda E. N. e MALAGRIS, Lúcia E. N. O stress emocional e seu tratamento. In: Rangé, Bernard. Psicoterapias cognitivo-comportamentais, Artmed, São Paulo, 2001.

LIPP, M. E. N. \& Novais, L. E. (2009). A Mágoa. In Lipp, M. (Org.). Sentimentos que causam stress: como lidar com eles (PP.159-167) (2. Ed.). Campinas: Papirus.

LlPP, Marilda Emmanuel Novaes. Manual do Inventário de Sintomas de Stress para Adultos de Lipp (ISSL) / Marilda Emmanuel Novaes Lipp. - São Paulo: Casa do Psicólogo, 2014.

MICHAELA, J. Mato Grosso terá estudo sobre estresse policial. Disponível em $<$ http:www.forumsegurança.org.br> Acesso em: 20 de outubro de 2011.

RODRIGUES, William Costa. Metodologia

Científica. FAETEC/IST. Paracambi, 2007.

Disponível em:

http://www.ebras.bio.br/autor/aulas/metodologia_cie ntifica.pdf $>$ Acesso em 7 de janeiro de 2016.

SELYE, H. (1984). Police stress._Police Stress Magazine, 1,_2-5.

KALIMO, R., El-Batawi, M. A. \& Cooper, C. L. 